\title{
INCIDENCE OF CAESAREAN WOUND INFECTION IN PATAN HOSPITAL, NEPAL
}

\author{
Pandit A*, Sharma P*, Yangzom K
}

\section{ABSTRACT}

This study aims to find the incidence and outcome of caesarean wound infection in Patan Hospital, during March 2002 to January 2003. We retrospectively analyzed records of patient who developed wound infection following caesarean section. Incidence of wound infection in Patan Hospital is comparatively low $2.76 \%$. The minor wound infections $(\mathbf{5 6 . 6 6 \%}$ ) required conservative management as an OPD basis while major wound infections required resuturing. The wound infection is more commonly associated with color of liqour, PROM and frequent number of vaginal examinations and emergency surgery.

\section{Key Words: Wound Infection, Caeserean Section, Nepal.}

\section{INTRODUCTION}

Caesarean Section is the most common surgical procedure in obstetrics practice. The Caesarean rate in different maternity units varies somewhere from $10 \%$ to $25 \%$ of total deliveries depending upon type of institutions, patient profile and various other factors. ${ }^{6,7}$ In Patan Hospital, the total number of deliveries during March 2002-2003 was 7,200 giving caesarean section rate of $17 \%$. Caesarean section accounts for $40 \%$ of major surgeries in Patan hospital, where general, urological, orthopedic and dental surgery are also done.

The post operative wound infection subjects patient to various physical and psychological distress. In a society where women play dominant role in household activities, her prolonged stay in hospital not only affects her but also her entire family.

In Nepal where the majority of the population is poor and, medical insurance facilities are within the research of very few fortunate ones, the prolonged hospital stay adds extrafinancial burden.

\footnotetext{
*Patan Hospital, Lalitpur, Nepal.
}

Address for correspondence :

Dr. Anil Pandit

Patan Hospital, Lalitpur, Nepal

Email: suresh@ntc.net.np

\section{OBJECTIVES OF THE STUDY}

The objectives of the study:

To estimate the incidence of post-operative caesarean wound infection in Patan Hospital

To study the outcome of the post-caesarean wound infection in Patan Hospital

\section{MATERIALS AND METHODS}

This is a hospital based study during March 2002 to January 2003. We retrospectively analyzed the records of the patient who developed post-operative caesarean wound infection in Patan Hospital during study period. There were total 1086 Caesarean sections performed and $1059(97.5 \%)$ patients came for follow up.

In our study we have defined major and minor wound infections are follows:

Major wound infection: A wound infection that discharges 
pus and may require secondary procedure. ${ }^{8}$

Minor wound infection: A wound infection that discharges pus but doesn't show any systemic response, discomfort or affect hospital stay. ${ }^{8}$

In Patan Hospital, patients are discharged on fourth postoperative day and first post-operative day is counted the day following surgery irrespective of time of surgery. The data were collected during the morning rounds from those patients who developed wound infection during hospital stay. The patients who developed wound infection following discharge from the hospital were picked up during follow up. Although the patients are advised to come for follow up after one week to Patan Hospital out patient department, we might have missed few cases who, didn't report to OPD and went to other private practitioners.

We estimated the incidence and outcome of post-operative caesarean section in relation to type of infection, type of management, duration of prolonged hospital stay. We also looked into the incidence of wound infection with age, Premature rupture of membrane (PROM), number of vaginal examination, color of liquor, indication of surgery and experience of the surgeons.

\section{RESULTS}

Incidence: There were total of 1086 caesarean section during study period. Out of which 30 patients developed either major or minor wound infection giving an incidence of $2.76 \%$.

PROM: Out of 30 cases, $10(33.33 \%)$ had premature rupture of membrane. 7 cases had rupture of membrane more than 24 hours. 2 cases had more than 6 hours. In 1 case it was only 3 hours duration. Out 7 who had rupture of membrane more than 24 hours, 4 developed major and 3 developed minor wound infections.

Fig. 1 : Premature rupture of membrane

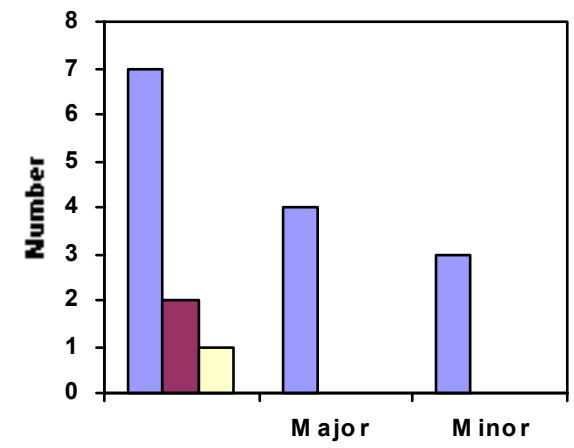

Number of per vaginal examination: In 14 cases, $\mathrm{p} \mathrm{v}$ examination was done less 2 times. But these cases were either associated with premature rupture of membrane or placenta previa where vaginal examinations are restricted or even contra-indicated. In 12 cases, $\mathrm{p} v$ examination was done 2-5 times and in four case $\mathrm{p} v$ examination was done more than 5 times.

Fig. 2 : Per Vaginal Examination

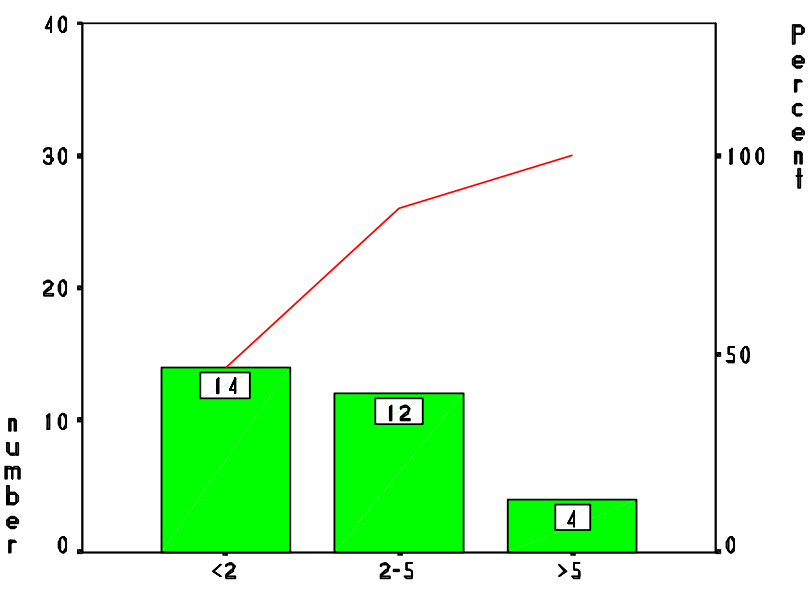

No. PV exam

LIQUOR COLOR: There were 18 cases with clear, 9 cases with moderate, 2 cases with light and 1 case with thick staining of liquor. Out of 18 cases with clear liquor, 6 cases had either premature rupture of membrane more than 24 hours or more than 3 number of $\mathrm{p} v$ examination.

Type of wound infection: There were $13(43.33 \%)$ major and $17(56.66 \%)$ minor wound infections.

Type of Management: 18 cases were managed conservatively while 12 required resuturing.

Fig. 3 : Duration of Hospital Stay

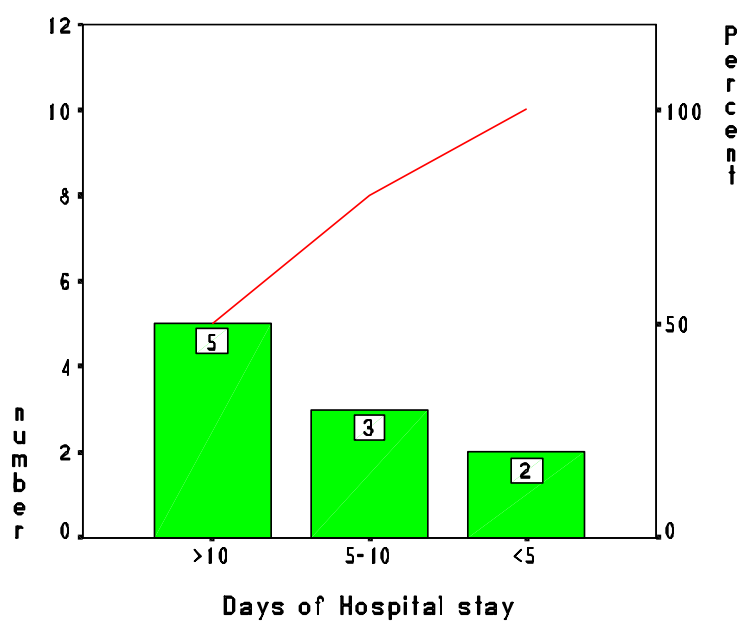


Duration of Hospital Stay: In 10 cases (33.33\%) hospital stay was prolonged. In 5 cases hospital stay was prolonged more than 10 days, in 3 cases between 5-10 days and in 2 cases less than 5 days.

Indication of surgery: In 26 cases the indication was emergency while 4 cases were elective.

\section{Level of Surgeons:}

Table I shows with increasing level of experience decreasing rate of infection. Incidence being highest among residents $(3.79 \%)$ and lowest among consultants ( $0.84 \%)$.

Relation of Major wound infection with liquor colour, PROM, and number of pv examination

There were 5 cases with moderate meconium and 8 cases of clear liquor who developed major wound infection. Those 8 cases with clear liquor, 6 cases either PROM more than 24 hours or more number of $\mathrm{p} v$ examination.

Thus major wound which required resuturing had either moderate meconium or PROM $>24$ hour or more number of pv examination done.

\section{Relation of Minor wound infection with liquor colour, PROM and number of pv examination.}

Out 17 cases of minor wound infection, 4 cases had moderate, 3 cases had light and 1 case had thick meconium. In 3 cases PROM more than 24 hours, 2 cases PROM more than 12 hours and in 1 cases less than 12 hours. In 5 cases pv examination was done more than 3 times. In case $\mathrm{p} v$ was done even 7 times, in 2 cases 6 times and in another 2 cases 4 times.

Thus minor wound infections had either light to thick meconium, PROM and more number of pv examination.

Organisms isolated: In only 5 cases, culture grew organisms. Out of which in 3 cases coagulase positive staphyloccocus, 2 cases enterobacter and 1 case bacillus species were isolated.

\section{DISCUSSION}

Several studies are conducted to estimate the incidence of postoperative wound infection in various part of world ranging from $<2 \%$ to $10 \%$. A multi-centric study done in United Kingdom, West Yorkshires, finds the incidence of $6.2 \%$ with the use of prophylactic antibioitcs. ${ }^{5}$ Another study done in Texas USA, found the incidence to be $7.8 \% .{ }^{4}$ It has also found that the subcutaneous skin thickness appears to be significant risk factor associated with abdominal wound infection after caesarean delivery. ${ }^{4}$ In Patan Hospital the incidence of wound infection is comparatively very low $(2.76 \%)$. This may be attributed to the fact that overall Nepalese population is less obese than western population although we didn't measure body mass index in our study.

Martens M G and associates. Texas 1995 have found that post caesarean wound infection is associated with rupture of membrane as less than 6 hour, emergency operation and morbid obesity. ${ }^{3}$ In our study 7 cases had rupture of membrane for more than 24 hours while 2 had more than 6 hours and 1 had 3 hours.

Labet A and et al, Mount Sinai, New York, March 2003 have found that meconium enhances the growth of perinatal pathogens and can occur as early as 6 hours of bacterial interaction with meconium ${ }^{2}$ Another study has found that even light meconium enhances the growth of Group B streptoccocus. ${ }^{1}$

In our study 5 cases of major wound infection had moderate meconium while 8 cases of minor wound infection had light to thick meconium.

\section{CONCLUSION}

The incidence of post caesarean wound infection in Patan Hospital is comparatively low $2.76 \%$ and they were minor wound infection $(56.66 \%)$ managed conservatively as an OPD basis while major wound infections required resuturing. 